\title{
Perfil das consultas de enfermagem ao paciente hematológico em hemocentro da
}

\section{região Norte do Brasil}

\author{
Profile of nursing consultations to hematological patients in a blood center in Northern Brazil \\ Perfil de las consultas de enfermería a pacientes hematológicos en un centro de sangre del Norte del
}

Brasil

Recebido: 16/03/2021 | Revisado: 23/03/2021 | Aceito: 28/03/2021 | Publicado: 06/04/2021

\author{
Thalyta Mayssa Paiva das Neves \\ ORCID: https://orcid.org/0000-0002-9960-7309 \\ Universidade do Estado do Pará, Brasil \\ E-mail: thatamayssa@yahoo.com.br \\ Ana Caroline Guedes Souza Martins \\ ORCID: https://orcid.org/0000-0001-7185-8520 \\ Universidade do Estado do Pará, Brasil \\ E-mail: carolguedes.devs@hotmail.com \\ Wesley Brandão Dias \\ ORCID: https://orcid.org/0000-0001-5033-9844 \\ Universidade Federal do Pará, Brasil \\ E-mail: wesleybrandao437@gmail.com \\ Maiza Silva de Sousa \\ ORCID: https://orcid.org/0000-0003-3816-8389 \\ Universidade do Estado do Pará, Brasil \\ E-mail: maizasousa1619@gmail.com \\ Gisele Maria Cardoso da Silva \\ ORCID: https://orcid.org/0000-0003-4202-2913 \\ Universidade Federal do Pará, Brasil \\ E-mail: gcardosomelo@gmail.br
}

\begin{abstract}
Resumo
Construção de um perfil assistencial voltado para a descrição da atuação do enfermeiro na Consulta de Enfermagem (CE) a pacientes hematológicos. Este estudo possui caráter quantitativo-descritivo, realizado em um hemocentro regional no município de Castanhal, localizado no Estado do Pará. Para a coleta de dados foi realizada uma análise dos protocolos operacionais padrão, verificando os registros de enfermagem e o levantamento das fichas de produção mensal de consultas de enfermagem, do período de junho a novembro de 2020. Foram analisadas as variáveis: quantitativo de pacientes cadastrados por diagnóstico; distribuição por faixa etária e sexo; tipos de doenças hematológicas; tipo de CE ( $1^{\mathrm{a}} \mathrm{vez}$, retorno e acolhimento); condutas nos desfechos das CE; E CE aos hemofílicos. Quanto aos pacientes cadastrados no hemocentro, há predomínio da Doença Falciforme (56,5\%) e Hemofilia A $(31,8 \%)$, na faixa etária de 0 a 10 anos, no sexo masculino. Quanto às CE nas doenças hematológicas $(\mathrm{n}=78)$ houve predomínio da Doença Falciforme (51,2\%) e Hemofilia A $(29,4 \%)$, com maior número de consultas pela primeira vez, que foram encaminhados para a Prioridade de Atendimento Hematológico. $\mathrm{Na} \mathrm{CE}$ ao hemofílicos predominou a profilaxia secundária. Os enfermeiros exercem um papel fundamental no atendimento ao paciente com doenças hematológicas e precisam estar adequadamente preparados para desempenhar esta responsabilidade, buscando a redução das distâncias entre a prática e o conhecimento científico disponível, de forma a ofertar um atendimento sistematizado e consequentemente, de qualidade.
\end{abstract}

Palavras-chave: Enfermagem no consultório; Cuidados de Enfermagem; Serviço de hemoterapia.

\begin{abstract}
Construction of a care profile aimed at describing the role of nurses in the Nursing Consultation (CE) to hematological patients. This study has a quantitative-descriptive character, carried out in a regional blood center in the municipality of Castanhal, located in the State of Pará. For data collection, an analysis of the standard operating protocols was performed, verifying the nursing records and the survey of the records monthly production of nursing consultations, from June to November 2020. The variables were analyzed: number of patients registered by diagnosis; distribution by age group and sex; types of hematological diseases; type of EC (1st time, return and reception); conducts in EC outcomes; And CE to hemophiliacs. Regarding the patients registered at the blood center, there is a predominance of sickle cell disease (56.5\%) and hemophilia A (31.8\%), in the age group of 0 to 10 years, in the male gender. As for FB in hematological diseases $(n=78)$, there was a predominance of sickle cell disease $(51.2 \%)$ and hemophilia A (29.4\%), with a greater number of consultations for the first time, which were referred to the
\end{abstract}


Hematological Care Priority. Secondary prophylaxis prevailed in EC for hemophiliacs. Nurses play a fundamental role in caring for patients with hematological diseases and need to be adequately prepared to perform this responsibility, seeking to reduce the distances between practice and available scientific knowledge, in order to offer systematic care and, consequently, to quality.

Keywords: Office nursing; Nursing care; Hemotherapy service.

\section{Resumen}

Construcción de un perfil de atención orientado a describir el rol del enfermero en la Consulta de Enfermería (CE) a los pacientes hematológicos. Este estudio tiene carácter cuantitativo-descriptivo, realizado en un centro regional de sangre en el municipio de Castanhal, ubicado en el Estado de Pará. Para la recolección de datos se realizó un análisis de los protocolos operativos estándar, verificando los registros de enfermería y la encuesta de producción de registros mensuales de consultas de enfermería, de junio a noviembre de 2020. Se analizaron las variables: número de pacientes registrados por diagnóstico; distribución por grupo de edad y sexo; tipos de enfermedades hematológicas; tipo de EC ( $1^{\mathrm{a}}$ vez, devolución y recepción); conductas en los resultados de la CE; Y EC a hemofílicos. En cuanto a los pacientes registrados en el centro de sangre, predomina la anemia falciforme $(56,5 \%)$ y la hemofilia A $(31,8 \%)$, en el grupo de edad de 0 a 10 años, en el sexo masculino. En cuanto a las CE en enfermedades hematológicas $(\mathrm{n}=78)$, hubo predominio de la enfermedad de células falciformes $(51,2 \%)$ y la hemofilia A $(29,4 \%)$, con un mayor número de consultas por primera vez, las cuales fueron derivadas a Atención Hematológica. Prioridad. En los hemofílicos predominó la profilaxis secundaria. El enfermero juega un papel fundamental en el cuidado de los pacientes con enfermedades hematológicas y necesita estar adecuadamente preparado para el desempeño de esta responsabilidad, buscando reducir las distancias entre la práctica y el conocimiento científico disponible, a fin de ofrecer una atención sistemática y, en consecuencia, de calidad.

Palabras clave: Enfermería de consulta; Atención de enfermería; Servicio de hemoterapia.

\section{Introdução}

O sangue sempre teve importância destacada na história, sendo empregado com finalidade terapêutica pelo homem há muitos séculos. Acreditava-se que a ingestão ou o banho de sangue poderiam curar certas doenças ou fortalecer o seu organismo. Essa prática caracterizava o período pré-histórico da transfusão no mundo. Com o passar do tempo, com o advento de novos conhecimentos e o avanço da ciência ocorreu o desenvolvimento da hemoterapia no mundo (Silva et al., 2020).

Atualmente, a hemoterapia consiste em uma das alternativas mais eficientes para o tratamento de patologias específicas e para a reposição de derivados sanguíneos e hemocomponentes (Dias, Cordeiro \& Gonçalves, 2021). O papel da enfermagem nos serviços de hemoterapia está consolidado em praticamente todo o ciclo do sangue, destacando-se a triagem de doadores, a assistência ao ato transfusional, assim como a assistência aos portadores de doenças hematológicas (Menezes et al., 2020).

No Brasil, os procedimentos hemoterápicos são regulamentados pelo Ministério da Saúde, através da Portaria $\mathrm{n}^{\circ} 158$ de 4 de fevereiro de 2016, em consonância com a Política Nacional de Sangue, Componentes e Derivados. Todos os serviços de hemoterapia do país devem funcionar de acordo com a Portaria citada e com os requisitos sanitários preconizados pela Agência Nacional de Vigilância Sanitária (ANVISA) (Brasil, 2016).

As atribuições dos enfermeiros e técnicos de enfermagem em hemoterapia são definidas através das Resoluções do Conselho Federal de enfermagem (COFEN) n 306, de 25 de abril de 2006 e nº 511, de 31 de março de 2016, atualizadas pela Resolução $n^{\text {o }}$ 629/2020, que instituem que a administração e a monitorização da infusão de hemocomponentes e hemoderivados são procedimentos de responsabilidade do enfermeiro, que poderão ser delegados ao técnico de enfermagem, desde que estejam devidamente capacitados (COFEN, 2020).

A partir da década de 60, no Brasil, a Consulta de Enfermagem (CE) vem sendo incorporada gradativamente em instituições de saúde pública e privada. Sua legalização ocorreu em 25 de junho de 1986 através da Lei n. $7.498 / 86$ que regulamentou o Exercício da enfermagem e estabeleceu essa atividade como privativa do Enfermeiro (Brasil, 1986). Em 1993, o Conselho Federal de Enfermagem, através da Resolução COFEN/159 estabeleceu a obrigatoriedade da realização da CE em todos os níveis de assistência à saúde (COFEN, 1993). 
Durante a CE o profissional utiliza-se de meios científicos para identificar situações de saúde/doença, objetivando a realização de prescrição e implementação de medidas de enfermagem que possam contribuir para promoção, prevenção, recuperação e reabilitação do indivíduo, da família e da comunidade. Para tanto a CE fundamenta-se nos princípios de universalidade, equidade, resolutividade e integralidade das ações de saúde (Jesus \& Carvalho, 1997).

Inúmeras foram as discussões quanto à necessidade de operacionalização dos referenciais teóricos de enfermagem, bem como os avanços em pesquisas fizeram surgir, na década de setenta, o Processo de Enfermagem. No que se refere ao portador de doença hematológica, é fundamental que sejam considerados os vários aspectos envolvidos, a exemplo: alterações físicas, psicológicas, emocionais e comportamentais, para ofertar uma assistência de enfermagem eficaz (Jesus \& Carvalho, 1997).

Os pacientes hematológicos apresentam problemas específicos, o que se exige um plano de cuidado próprio para esta demanda. Dessa forma, cabe à enfermagem utilizar recursos possíveis de aprimorar a assistência prestada a esses pacientes, com implementação de intervenções de enfermagem que possibilitem a melhoria da qualidade dos resultados esperados. Portanto, é indispensável o preparo do enfermeiro, bem como dos familiares que participam do processo de assistência a pessoa com alterações hematológicas (Honório \& Caetano, 2009).

A enfermagem está inserida na prática em hemoterapia desde a antiguidade, desenvolvida pelos profissionais envolvidos no cuidado, tanto ao doador de sangue quanto ao receptor de componentes sanguíneos. Porém, somente em 1990 que os profissionais, em eventos científicos, discutiram sobre a lacuna existente na atuação junto a doadores e pacientes hematológicos, deixando evidente a necessidade de regulamentação das atividades desenvolvidas (Frantz, 2018).

A Fundação Centro de Hemoterapia e Hematologia do Pará (HEMOPA) é referência estadual no diagnóstico e tratamento das patologias ligadas ao sangue. Porém, são escassos os estudos que remetem a temática CE em hemocentros, portanto não foi encontrado nenhum trabalho que descreva a realização da $\mathrm{CE}$ aos usuários assistidos em Centro de Hemoterapia e Hematologia na região Norte, no Brasil e no mundo, o que despertou o interesse em explorar e descrever sobre o assunto.

Nesse contexto, o presente estudo objetiva a construção de um perfil assistencial voltado para a descrição da atuação do enfermeiro na CE a pacientes hematológicos.

\section{Metodologia}

Este estudo possui caráter quantitativo-descritivo, sendo a pesquisa quantitativa aquela em que se coletam e analisam dados e variáveis. Dessa forma, este tipo de pesquisa é capaz de identificar a natureza profunda das realidades, seu sistema de relações, sua estrutura dinâmica. Ela também pode determinar a força de associação ou correlação entre variáveis, a generalização e objetivação dos resultados através de uma mostra que faz inferência a uma população. Além do estudo da associação ou correlação, também pode, ao seu tempo, fazer inferências causais que explicam por que as coisas acontecem ou não de uma forma determinada (Esperón, 2017).

O estudo foi realizado no Hemocentro Regional de Castanhal (HRC), localizado na cidade de Castanhal, que fica a 72 km da capital Belém, Pará. Está vinculado ao Hemocentro coordenador da Fundação Centro de Hemoterapia e Hematologia do Pará (HEMOPA), constituindo a hemorrede estadual. É responsável pela área de gestão II que corresponde a 04 regiões de integração: 1- Região do Guamá; 2- Região do Tocantins; 3- Região do Rio Capim e 4- Região do Rio Caeté.

Para a coleta de dados foi realizada uma análise dos Protocolos Operacionais Padrão (POP), verificando os registros de enfermagem e o levantamento das fichas de produção mensal de CE, do período de junho a novembro de 2020.

As informações foram colhidas por meio da estatística mensal de atendimentos de enfermagem e analisou as seguintes variáveis: número de atendimentos, diagnóstico dos pacientes atendidos, tipo de atendimento e categoria do atendimento. 
Foram organizados em um banco de dados e tabulados no Programa Microsoft Excel através de estatística descritiva, para posterior análise crítica.

Os dados pesquisados foram adquiridos através da tabulação condensada dos indicadores de enfermagem, portanto não se utilizou prontuários. Em decorrência disso, o estudo não necessitou do parecer de um Comitê de Ética em Pesquisa. Todos os princípios éticos foram seguidos, conforme as recomendações nacionais e internacionais de pesquisa, e ainda, vale ressaltar que houve a liberação da instituição proponente, através do Núcleo de pesquisa do HEMOPA (NEPES).

\section{Resultados}

O Quadro 1 representa o quantitativo de pacientes cadastrados no HRC de acordo com os seus diagnósticos estudados, no qual destaca-se o predomínio da Doença Falciforme (56,5\%) e Hemofilia A (31,8\%).

Quadro 1 - Distribuição do quantitativo de pacientes cadastrados por diagnóstico estudado, HEMOPA, Castanhal, 2020.

\begin{tabular}{ccccccc}
\hline Diagnóstico & $\begin{array}{c}\text { Hemofilia } \\
\text { A }\end{array}$ & $\begin{array}{c}\text { Hemofilia } \\
\text { B }\end{array}$ & $\begin{array}{c}\text { Doença de Von } \\
\text { Willebrand }\end{array}$ & $\begin{array}{c}\text { Doença } \\
\text { falciforme }\end{array}$ & Talassemia & TOTAL \\
\hline $\begin{array}{c}\mathbf{N}^{\mathbf{0}} \text { de pacientes } \\
\text { cadastrados }\end{array}$ & 22 & 02 & 05 & 39 & 01 & $\mathbf{6 9}$ \\
\hline
\end{tabular}

Fonte: HEMOPA (2020).

Observa-se no Quadro 2 que as faixas etárias são variadas nas CE e que nos meses de setembro e outubro houve um discreto aumento no número de pessoas atendidas, com destaque para o atendimento pediátrico, no qual observou-se que a maioria das consultas de enfermagem ocorreram na faixa etária de 0 a 10 anos. Ressalta-se que não houve nenhum atendimento para maiores de 60 anos com diagnóstico definido de hemoglobinopatias e/ou coagulopatias.

Quadro 2 - Distribuição por Faixa Etária, Consulta de Enfermagem, HEMOPA, Castanhal, junho a novembro de 2020.

\begin{tabular}{lrrrrrrrr}
\hline Meses/Faixa etária & $\mathbf{0}$ a 10 & $\mathbf{1 1}$ a 20 & $\mathbf{2 1}$ a 30 & $\mathbf{3 1}$ a 40 & $\mathbf{4 1}$ a 50 & $\mathbf{5 1}$ a 60 & mais de 60 & TOTAL \\
\hline Junho & 1 & 1 & 0 & 4 & 1 & 0 & 0 & $\mathbf{7}$ \\
\hline Julho & 3 & 5 & 2 & 1 & 1 & 1 & 0 & $\mathbf{1 3}$ \\
\hline Agosto & 6 & 4 & 1 & 0 & 0 & 2 & 0 & $\mathbf{1 3}$ \\
\hline Setembro & 4 & 3 & 1 & 4 & 2 & 1 & 0 & $\mathbf{1 5}$ \\
\hline Outubro & 7 & 3 & 1 & 2 & 2 & 1 & 0 & $\mathbf{1 6}$ \\
\hline Novembro & 3 & 2 & 0 & 7 & 0 & 2 & 0 & $\mathbf{1 4}$ \\
\hline TOTAL & 24 & 18 & 5 & 18 & 6 & 7 & 0 & $\mathbf{7 8}$ \\
\hline
\end{tabular}

Fonte: HEMOPA (2020).

Constatou-se que nos meses de junho e julho predominaram atendimentos à pacientes do sexo feminino, enquanto que a partir de agosto até novembro predominou no sexo masculino. De forma geral os pacientes do sexo masculino compareceram com mais frequência às CE (Gráfico 1). 
Gráfico 1 - Distribuição por sexo, Consulta de enfermagem, HEMOPA, Castanhal, junho a novembro, 2020.

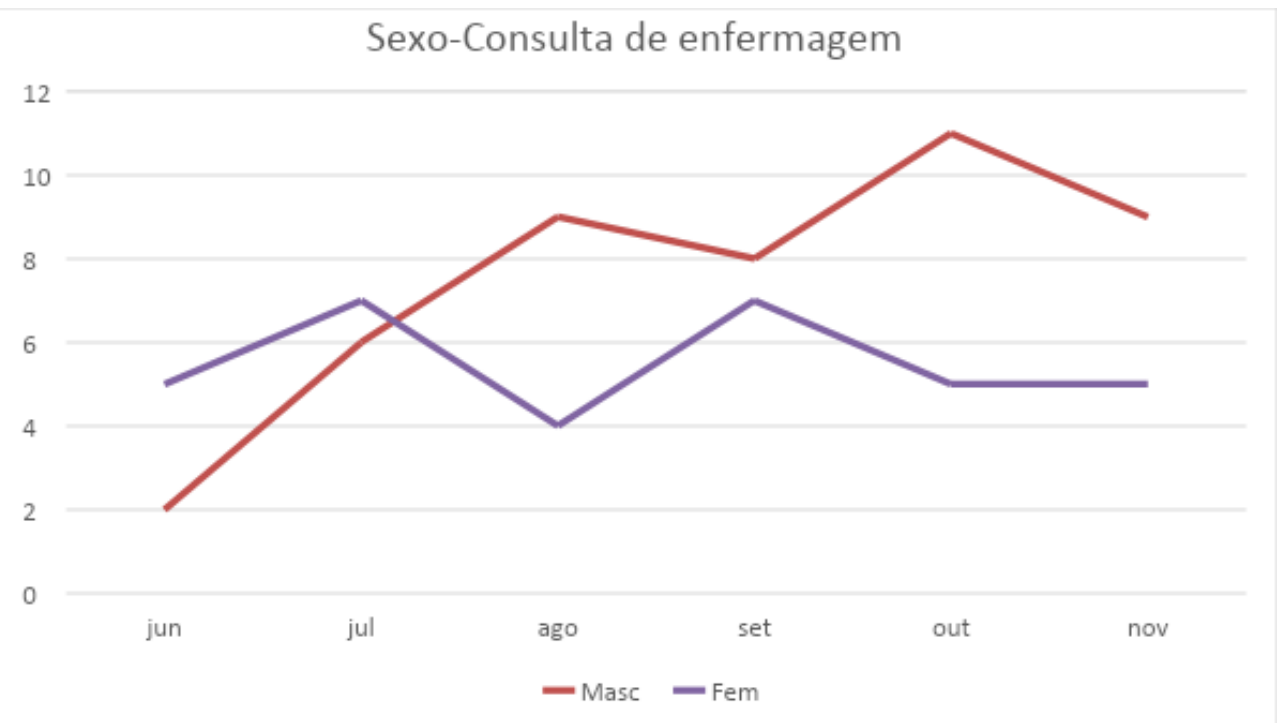

Fonte: HEMOPA (2020).

$\mathrm{Na}$ Tabela 1 estão representadas as doenças hematológicas predominantes nas $\mathrm{CE}(\mathrm{n}=78)$ realizadas no período estudado, em que houve predomínio da Doença Falciforme (51,2\%), seguido de Hemofilia A $(29,4 \%)$.

Tabela 1 - Doenças Hematológicas, Consulta de enfermagem, HEMOPA, Castanhal, junho a novembro, 2020.

\section{DOENÇAS HEMATOLÓGICAS-CONSULTA DE ENFERMAGEM}

\begin{tabular}{lccccccc}
\hline & JUN & JUL & AGO & SET & OUT & NOV & TOTAL \\
\hline COAGULOPATIAS & & & & & & & \\
\hline Hemofilia A & 2 & 3 & 4 & 4 & 4 & 6 & $\mathbf{2 3}$ \\
\hline Hemofilia B & 0 & 0 & 0 & 0 & 0 & 0 & $\mathbf{0 0}$ \\
\hline DVW & 1 & 2 & 1 & 1 & 0 & 0 & $\mathbf{0 5}$ \\
\hline Outras Coagulopatias & 0 & 2 & 0 & 0 & 0 & 0 & $\mathbf{0 2}$ \\
\hline TOTAL & 3 & 7 & 5 & 5 & 4 & 6 & $\mathbf{3 0}$ \\
\hline HEMOGLOBINOPATIAS & & & & & & & \\
\hline D.Falciforme & 4 & 4 & 8 & 7 & 10 & 7 & $\mathbf{4 0}$ \\
\hline Talassemias & 0 & 1 & 0 & 1 & 0 & 1 & $\mathbf{0 3}$ \\
\hline Outras Anemias & 0 & 1 & 0 & 2 & 2 & 0 & $\mathbf{0 5}$ \\
\hline TOTAL & 4 & 6 & 8 & 10 & 12 & 8 & $\mathbf{4 8}$ \\
\hline
\end{tabular}

Fonte: HEMOPA (2020)

Nas consultas de enfermagem, houve maior número de consultas pela primeira vez, seguido por consultas de acolhimento. É necessário esclarecer que a classificação de primeira vez foi adotada neste estudo para representar os pacientes que estavam sendo assistidos pela primeira vez na CE e não aqueles pacientes inseridos pela vez nos demais serviços do hemocentro. No Gráfico 2 está representado o tipo de CE realizada no período estudado. 
Gráfico 2 - Tipo de consulta, Consulta de enfermagem, HEMOPA, Castanhal, junho a novembro, 2020.

\section{Tipo de consulta de enfermagem}

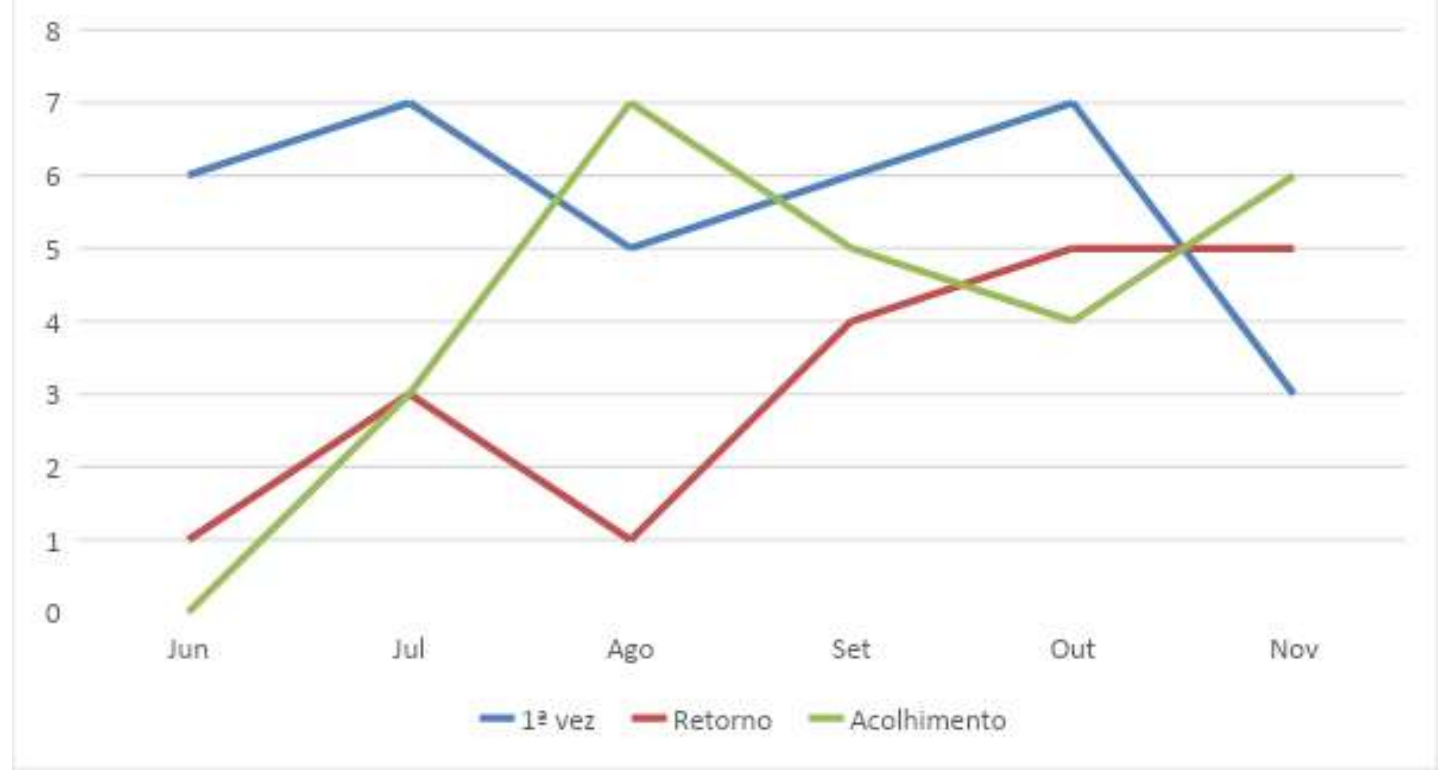

Fonte: HEMOPA (2020).

Quanto às condutas, nos desfechos das consultas de enfermagem, há predomínio de encaminhamento do usuário para a Prioridade de Atendimento Hematológico (PAH), seguindo pelo agendamento médico das consultas subsequentes.

Tabela 2 - Conduta, Consulta de enfermagem, HEMOPA, Castanhal, junho a novembro, 2020.

\begin{tabular}{ccccccccc}
\hline \multicolumn{7}{c}{ CONDUTA-CONSULTA DE ENFERMAGEM } \\
\hline & JUN & JUL & AGO & SET & OUT & NOV & TOTAL \\
\hline PAH Médico & 5 & 13 & 13 & 14 & 15 & 14 & $\mathbf{7 4}$ \\
\hline Agendamento Médico & 2 & 0 & 0 & 1 & 1 & 0 & $\mathbf{0 4}$ \\
\hline TOTAL & 7 & 13 & 13 & 15 & 16 & 14 & $\mathbf{7 8}$
\end{tabular}

Fonte: HEMOPA (2020).

Na Tabela 3 estão representadas as consultas de enfermagem aos hemofílicos atendidos no período estudado, em que houve predomínio de profilaxia secundária. 
Tabela 3 - Hemofílicos atendidos em programa, Consulta de enfermagem, HEMOPA, Castanhal, junho a novembro, 2020.

\begin{tabular}{lccccccc}
\hline \multicolumn{7}{c}{ HEMOFÍLICOS-CONSULTA DE ENFERMAGEM } \\
\hline & JUN & JUL & AGO & SET & OUT & NOV & TOTAL \\
\hline Profilaxia primária & 0 & 0 & 0 & 0 & 1 & 0 & $\mathbf{0 1}$ \\
\hline Profilaxia secundária & 2 & 3 & 2 & 4 & 3 & 5 & $\mathbf{1 9}$ \\
\hline Imunotolerância & 0 & 0 & 0 & 0 & 0 & 0 & $\mathbf{0 0}$ \\
\hline Demanda & 0 & 0 & 0 & 0 & 0 & 0 & $\mathbf{0 0}$ \\
\hline MYPKFIT & 0 & 0 & 0 & 0 & 0 & 0 & $\mathbf{0 0}$ \\
\hline TOTAL & 2 & 3 & 2 & 4 & 4 & 6 & $\mathbf{2 0}$ \\
\hline
\end{tabular}

Fonte: HEMOPA (2020).

\section{Discussão}

$\mathrm{Na} \mathrm{CE}$ observou-se o predomínio do atendimento ao paciente do sexo masculino e a faixa etária que prevaleceu foi de 0 a 10 anos. Resultado semelhante foi retratado no estudo de Jesus e Carvalho (1997), no qual os dados revelam que a população estudada, quanto à variável sexo, houve uma distribuição ligeiramente maior para o sexo masculino comparado ao sexo feminino. Quanto à variável idade, houve uma discordância em relação ao estudo, pois a distribuição foi predominante para a faixa etária classificada de 18 a 59 anos.

Pessoas com hemoglobinopatias como na Doença Falciforme (DF) foram as que mais procuraram os serviços de enfermagem. A DF é uma das hemoglobinopatias hereditárias mais comuns em todo o mundo e compreende um termo genérico que engloba um grupo de anemias hemolíticas hereditárias (Veras et al., 2020). Apesar das características marcadamente distintas das DF, suas manifestações clínicas e hematológicas são semelhantes (Brasil, 2014). A anemia falciforme (AF) é a doença genética com apresentação clínica e hematológica mais grave e também a de maior prevalência. No Brasil, a AF é altamente prevalente e considerada um problema de saúde pública (De Sousa \& Silva, 2017). Além disso, é caracterizada por diversas complicações agudas e crônicas, como anemia, infecções graves, crises hemolíticas e vasooclusivas, crises de dor recorrente, acidente vascular cerebral, síndrome torácica aguda, hipertensão pulmonar e danos crônicos a órgãos, o que requer um cuidado continuado e orientações específicas de enfermagem (Veras et al., 2020).

Essa realidade vem sendo contextualizada por Kikuchi (2007) ao mencionar que a DF possui complicações clínicas de níveis que variam de um estado de bem-estar a um estado de urgência e emergência. De acordo com o nível de complexidade a DF, a sua resolução deve ser mediada pelos centros em hemoterapia.

Prevaleceram os atendimentos de primeira vez, sendo este classificado como o primeiro atendimento do paciente na $\mathrm{CE}$, o que demonstra que os pacientes cadastrados passaram a procurar mais pelo referido serviço, não apenas pelo trabalho que as enfermeiras vêm desenvolvendo na sensibilização da importância deste serviço no acompanhamento de pacientes, como a disseminação da informação da existência do mesmo pela equipe multidisciplinar, sobretudo pelos encaminhamentos médicos junto aos pacientes com diagnóstico definido (hemoglobinopatias e coagulopatias).

Durante o período de atuação da pesquisadora foi perceptível que a $\mathrm{CE}$, tanto para a instituição quanto para o paciente, precisa ser divulgada e esclarecida. Muitos ainda a percebem apenas como uma triagem ou uma etapa do protocolo que precisa ser seguido. Faz-se necessário propagar os resultados dessa pesquisa e disseminar o significado da CE ao paciente e familiares de pessoas com doenças hematológicas, assim como ratificar o fluxo de atendimento da enfermagem no hemocentro. 
A pesquisa de Jesus e Carvalho (1997) voltada para a utilização do Diagnóstico de Enfermagem em pacientes portadores de doenças hematológicas hospitalizadas concluiu que para o enfermeiro identificar perfis diagnósticos de diferentes clientelas, é fundamental que domine o processo de julgamento clínico. Nesse processo, o enfermeiro avalia o significado das informações sobre o cliente, estabelece relações entre os dados, nomeia o fenômeno e chegam ao diagnóstico a partir da inferência e de estratégias de pensamento, com base em suas experiências, conhecimentos e valores. Requerem habilidades de manipulação dos dados, domínio de conhecimento, aptidão intelectual, objetividade, pensamento crítico, experiência, intuição e tomada de decisão.

Os achados nesse estudo, associados à discussão de Amaral et al. (2016) revela que por mais que o profissional de enfermagem seja requisitado para desenvolver suas ações de saúde no contexto da hemoterapia, o mesmo não possui o reconhecimento de sua categoria, tornando o profissional desconhecedor da importância e complexidade do serviço hemoterápico. Esse desconhecimento pode ser resultado da formação acadêmica deste profissional, onde não se enfatizou os serviços assistenciais em hemoterapia.

Além disso, Torres et al. (2021) afirma que a preparação dos acadêmicos de enfermagem quanto à hemoterapia durante a graduação é deficitária. Constatou-se que 63,2\% relataram terem assistido palestras e/ou participado de visitas técnicas a algum serviço de hemoterapia e apenas 18,4\% afirmaram terem tido as aulas sobre a temática.

Ademais, isto se tornou evidente ao longo desta pesquisa pela escassez de publicações referentes ao tema que retratam a CE diretamente ao paciente hematológico. Desta forma, neste estudo evidenciou-se que o enfermeiro de Centro de Hemoterapia devidamente capacitado pode assistir o paciente com diagnóstico hematológico e realizar com êxito a CE.

\section{Considerações Finais}

Em resumo, é perceptível a necessidade e importância do profissional de enfermagem no atendimento a pessoas com doenças hematológicas. Antes, o enfermeiro exercia a profissão de forma empírica, hoje, vivencia uma nova realidade adaptada à busca de conhecimento científico, conquistando espaços diversificados na área da saúde. Refere-se a atuação desses profissionais como membro fundamental da equipe multidisciplinar nos centros de hemoterapia permitindo-lhes assumir, dentro da legalidade, novas funções e cargos.

Com o avanço do conhecimento técnico científico da enfermagem nos últimos anos sobre a hemoterapia, ainda é necessário investir em mais publicações nessa área, visto que pesquisas que fomentem a prática da enfermagem baseada em evidências em centros de hemoterapia ainda é escassa. A publicação científica nessa área pode ajudar na consolidação da enfermagem na especialidade quanto aos cuidados em serviços de hemoterapia, tornando assim a profissão uma das protagonistas na rede dos serviços hemoterápicos.

Outrossim, estudos futuros podem ser realizados para se conhecer a realidade das consultas de enfermagem em hemocentros de outros Estados e regiões do Brasil, assim como estudos para estruturação da Sistematização da Assistência de Enfermagem com vistas a melhorar a organização dos serviços e a qualidade da atenção à saúde prestada a este público.

\section{Referências}

Amaral, J. H. S., et al. (2016). Hemoterapia: um desafio no cotidiano da equipe de enfermagem. Rev. Enferm. UFPE on line Recife, $10(6)$ : $4820-7$.

Brasil. (1986). Lei nº 7.498/86, 25 de Junho de 1986. Dispõe sobre a regulamentação do exercício da Enfermagem e dá outras providências. Diário Oficial da União. Brasília.

Brasil. (2014). Doença falciforme: saúde bucal: prevenção e cuidado. Ministério da Saúde.

Brasil. (2016). Ministério da Saúde. Portaria n ${ }^{\circ}$ 158, de 4 de fevereiro de 2016. Redefine o regulamento técnico de procedimentos hemoterápicos.

Conselho Regional de Enfermagem (COFEN). (1993). Resolução n 159/1993. Consulta de Enfermagem. Conselho Federal de Enfermagem. 
Research, Society and Development, v. 10, n. 4, e20310413948, 2021

(CC BY 4.0) | ISSN 2525-3409 | DOI: http://dx.doi.org/10.33448/rsd-v10i4.13948

Conselho Regional de Enfermagem (COFEN). (2020). Resolução COFEN nº 629/2020. Aprova e Atualiza a Norma Técnica que dispõe sobre a Atuação de Enfermeiro e de Técnico de Enfermagem em Hemoterapia.Brasília: Conselho Federal de Enfermagem.

Da Silva, H. R. et al. (2020). Onco-hematologia: perspectivas e avanços. Research, Society and Development, 9(8), e269985684-e269985684.

De Sousa, A. M., \& Silva, F. R. A. (2017). Sickle cell trait in Brazil: literature review and information technology proposal for basic heath providers' guidance. Revista de Medicina da UFC, 57 (2), 37-43.

Dias, M. M., Cordeiro, B. C., Gonçalves, G. F. (2021). Educação permanente aplicada aos profissionais do setor de hemoterapia: uma revisão integrativa. Research, Society and Development, 10(1), e38510111818-e38510111818.

Esperón, J. M. T. (2017). Pesquisa quantitativa na ciência da enfermagem. Esc. Anna Nery. 21(1): e20170027.

Frantz, S. R. S. (2018). Processo de trabalho dos enfermeiros no Serviço de hemoterapia: entre o prescrito e o real. [Tese de Doutorado]. Florianópolis: Universidade Federal de Santa Catarina, Centro de Ciências da Saúde, Programa de Pós-Graduação em Enfermagem.

Honório, R. P. P., Caetano, J.A. (2009). Elaboração de um protocolo de assistência de enfermagem ao paciente hematológico: relato de experiência. Rev. Eletr. Enf. 11(1): 188-93.

Jesus, C. A. C., Carvalho, E. C. (1997). Diagnósticos de enfermagem em clientes com alterações hematológicas: uso da Taxonomia I da NANDA. Rev. LatinoAm. Enfermagem 5(4): 91-99.

Kikuchi, B. A. (2007). Assistência de enfermagem na doença falciforme nos serviços de atenção básica. Rev. Bras. Hematol. Hemoter 29(3): 331-338.

Menezes, R, A. et al. (2020). Inaptidão clínica e sorológica entre doadores de sangue em um serviço de hemoterapia. Research, Society and Development, 9(10), e2659108486-e2659108486.

Torres, R. C.et al. (2021). Atuação do enfermeiro em hemoterapia: a visão do formando. Brazilian Journal of Development, 7(2), $16000-16014$.

Veras, G. A. R. et al. (2020). Health-related quality of life in children and adolescents with sickle cell disease. Research, Society and Development, 9(9), e998998120-e998998120 